CRYSTALLOGRAPHIC COMMUNICATIONS

ISSN 2056-9890

Received 6 March 2018

Accepted 13 April 2018

Edited by L. Fabian, University of East Anglia, England

Keywords: crystal structure; lamotrigine; ethanolate.

CCDC reference: 1826282

Supporting information: this article has supporting information at journals.iucr.org/e

\section{Lamotrigine ethanol monosolvate}

\author{
Charlie L. Hall, Jason Potticary, Hazel A. Sparkes, Natalie E. Pridmore and Simon R.
} Hall*

School of Chemistry, University of Bristol, Cantock's Close, Bristol, England BS8 1TS, England. *Correspondence e-mail: simon.hall@bristol.ac.uk

Lamotrigine is an active pharmaceutical ingredient used as a treatment for epilepsy and psychiatric disorders. Single crystals of an ethanolate solvate, $\mathrm{C}_{9} \mathrm{H}_{7} \mathrm{Cl}_{2} \mathrm{~N}_{5} \cdot \mathrm{C}_{2} \mathrm{H}_{5} \mathrm{OH}$, were produced by slow evaporation of a saturated solution from anhydrous ethanol. Within the crystal structure, the lamotrigine molecules form dimers through $\mathrm{N}-\mathrm{H} \cdots \mathrm{N}$ hydrogen bonds involving the amine $\mathrm{N}$ atoms in the ortho position of the triazine group. These dimers are linked into a tape motif through hydrogen bonds involving the amine $\mathrm{N}$ atoms in the para position. The ethanol and lamotrigine are present in a 1:1 ratio in the lattice with the ethyl group of the ethanol molecule exhibiting disorder with an occupancy ratio of 0.516 (14):0.484 (14).

\section{Chemical context}

Anticonvulsants are a group of drugs used principally in the treatment of epilepsy, which have also been shown to aid in the treatment of psychiatric conditions such as bipolar disorder. Although the drugs are effective when inside the body, many suffer from having low solubility and bioavailability. Prime examples of such drugs are carbamazepine (Uzunović et al., 2010), phenytoin (Widanapathirana et al., 2015) and lamotrigine (Vaithianathan et al., 2015), which are all categorised as BCS (biopharmaceutical classification system) class II (low solubility, high permeability).

In an attempt to increase the solubility of BCS class II drugs, extensive studies have been undertaken to produce crystal structures including the active pharmaceutical ingredients (APIs) with lower crystal lattice energies. In the case of lamotrigine, Cheney et al. (2010) investigated the solubility of 10 novel forms, including salts, co-crystals and solvates, showing the possibility of creating many stable lamotrigine compounds. The structures of lamotrigine co-crystals and solvates are stabilized due to the large number of hydrogen bonds that can form with the 1,2,4-triazine-3,5-diamine group.<smiles>Nc1nnc(-c2cccc(Cl)c2Cl)c(N)n1</smiles>

In this work, the structure for the ethanolate (I), previously only obtained as a powder pattern (Garti et al., 2008), is defined. This new structure determination affords a deeper 
Table 1

Chosen parameters for the comparison of lamotrigine alcohol solvates.

\begin{tabular}{llll}
\hline Structure & Central dihedral angle $\left(^{\circ}\right)$ & Dimerization motif & Density $\left(\mathrm{g} \mathrm{cm}^{-1}\right)$ \\
\hline Methanol disolvate & $63.7(2)$ & para & 1.50 \\
Ethanol monohydrate & $67.6(0)$ & para & 1.49 \\
Methanol monosolvate & $80.1(5)$ & ortho & 1.45 \\
Ethanol solvate (I) & $63.5(9)$ & ortho & 1.42 \\
2-Propanol solvate & $69.6(8)$ & ortho & 1.36 \\
Butan-1-ol solvate monohydrate & $71.2(1)$ & para & 1.34 \\
\hline
\end{tabular}

insight into the different hydrogen-bonding networks that can form in the lamotrigine crystal.

\section{Structural commentary}

A displacement ellipsoid plot for lamotrigine ethanolate is shown in Fig. 1. The central dihedral, $\mathrm{C} 1-\mathrm{C} 6-\mathrm{C} 7-\mathrm{C} 8$, sits at an angle of $63.5(9)^{\circ}$, the flexibility of which allows for the inclusion of solvent molecules to form hydrogen-bonding networks. Central dihedral angles for lamotrigine solvates are included in Table 1. Fig. 2 shows the unit cell for (I), which consists of eight lamotrigine molecules and eight ethanol molecules. The main motif within the structure is a lamotrigine dimer stabilized by two ethanol molecules. Here the lamotrigine dimer forms using the amine $\mathrm{N}$ atoms in the ortho position of the triazine group.

\section{Supramolecular features}

In the crystal, adjacent in-plane lamotrigine dimers are linked via hydrogen bonding of the amines in the para position of the triazine group (Table 2). Each dimer sits at an angle of $67.2(5)^{\circ}$ to the next closest dimer, measured with respect to the in-plane triazine rings, highlighted in Fig. 3.

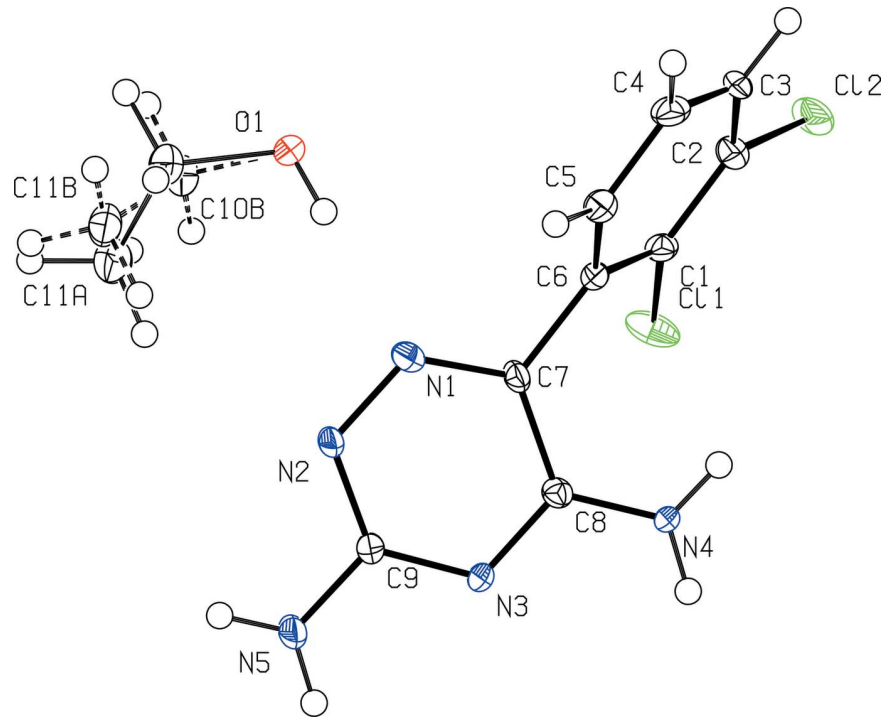

Figure 1

A displacement ellipsoid plot of (I), showing the atom-labelling scheme. Displacement ellipsoids are drawn at the $50 \%$ probability level.

\section{Database survey}

A database survey of the Cambridge Structural Database (CSD, version 5.38, last update May 2017; Groom et al., 2016) showed a list of 35 existing co-crystal/solvate structures for lamotrigine, including 6 structures incorporating alcohols, but no ethanol solvate. The most similar structure compositionally to (I) is the ethanol solvate monohydrate (Cheney et al., 2010); however, the arrangement contrasts quite dramatically, with

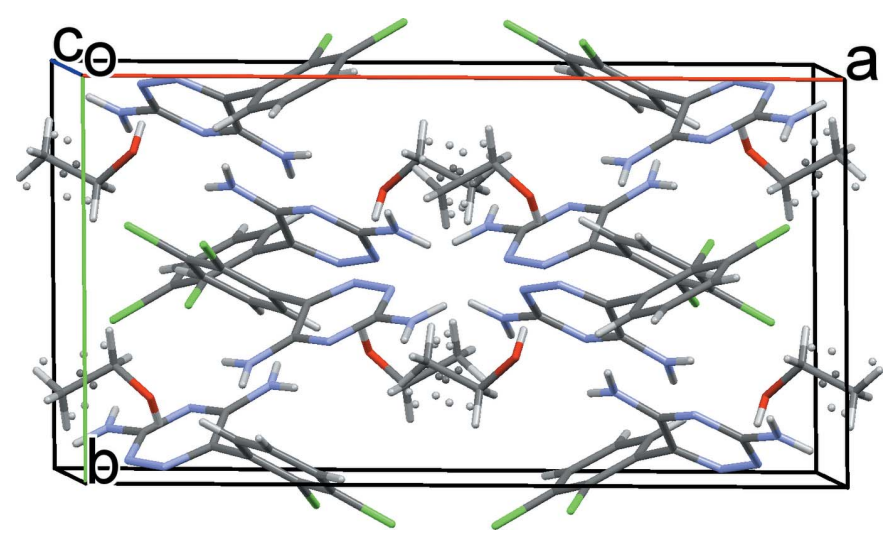

Figure 2

The crystal packing of (I), viewed along the $c$ axis.

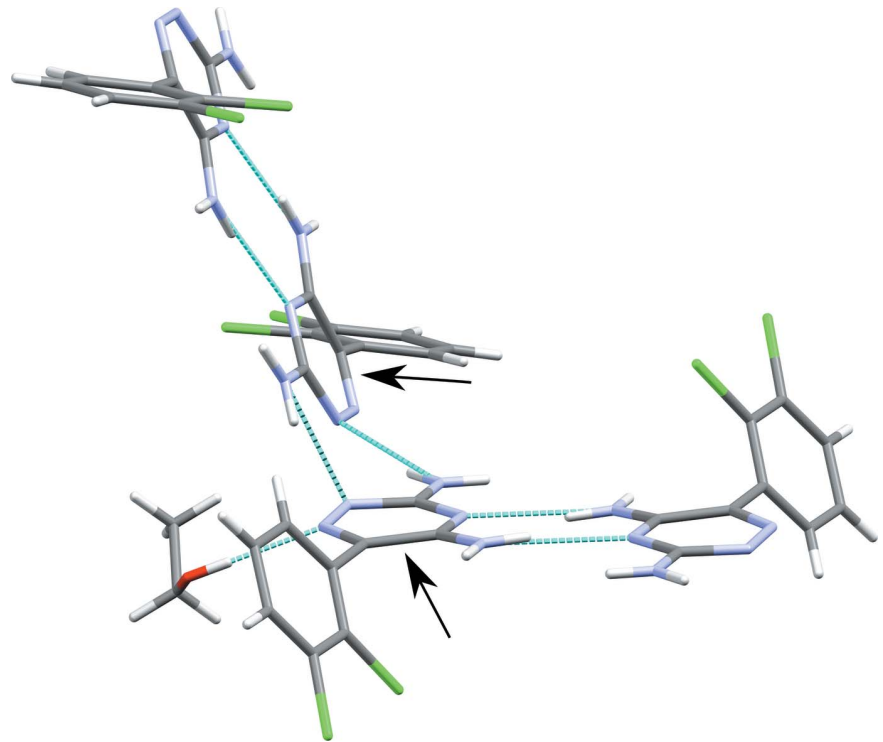

Figure 3

The bonding motif of adjacent lamotrigine dimers. The angle between the dimers was calculated using the planes of the indicated triazine rings. 
Table 2

Hydrogen-bond geometry $\left(\AA,^{\circ}\right)$.

\begin{tabular}{lllll}
\hline$D-\mathrm{H} \cdots A$ & $D-\mathrm{H}$ & $\mathrm{H} \cdots A$ & $D \cdots A$ & $D-\mathrm{H} \cdots A$ \\
\hline $\mathrm{O} 1-\mathrm{H} 1 A \cdots \mathrm{N} 1$ & 0.84 & 2.01 & $2.848(7)$ & 179 \\
$\mathrm{~N} 4-\mathrm{H} 4 A \cdots \mathrm{N} 3^{\mathrm{i}}$ & 0.88 & 2.10 & $2.972(7)$ & 172 \\
$\mathrm{~N}^{\mathrm{i}}-\mathrm{H} 4 B \cdots \mathrm{O} 1^{i i}$ & 0.88 & 2.14 & $2.841(7)$ & 137 \\
$\mathrm{~N}^{\mathrm{ii}}-\mathrm{H} 5 A \cdots \mathrm{O} 1^{\mathrm{iii}}$ & 0.88 & 2.16 & $3.014(7)$ & 163 \\
$\mathrm{~N} 5-\mathrm{H} 5 B \cdots \mathrm{N} 2^{\text {iv }}$ & 0.88 & 2.14 & $2.987(8)$ & 161
\end{tabular}

Symmetry codes: (i) $-x+\frac{1}{2},-y+\frac{3}{2},-z$; (ii) $-x+\frac{1}{2}, y+\frac{1}{2},-z+\frac{1}{2}$; (iii) $x,-y+1, z-\frac{1}{2}$; (iv) $-x+1, y,-z+\frac{1}{2}$.

the dimer formation of the lamotrigine molecules using the amine $\mathrm{N}$ atoms in the para position, shown in Fig. 4. This change in dimerization motif leads to a reduction in density of the lamotrigine ethanolate over the lamotrigine ethanol monohydrate by $5 \%$.

Analysis of the previously published lamotrigine alcohol solvates shows a trend between the alcohol chain length and whether the lamotrigine dimers form on the ortho or para group of the triazine. The two densest structures are the methanol disolvate (Hanna et al., 2009) and the ethanol solvate monohydrate, where lamotrigine dimers are connected via the amines in the para position of the triazine. Conversely, the methanol monosolvate (Janes et al., 1989), isopropanol solvate (Qian et al., 2009) and title compound form dimers from the amine on the ortho positions. The least dense structure is the butan-1-ol solvate monohydrate (Sridhar \& Ravikumar, 2011), which has similar arrangement to the dense structures, with the dimers held apart by the large butanol solvent molecules. The densities of the lamotrigine structures are highlighted in Table 1.

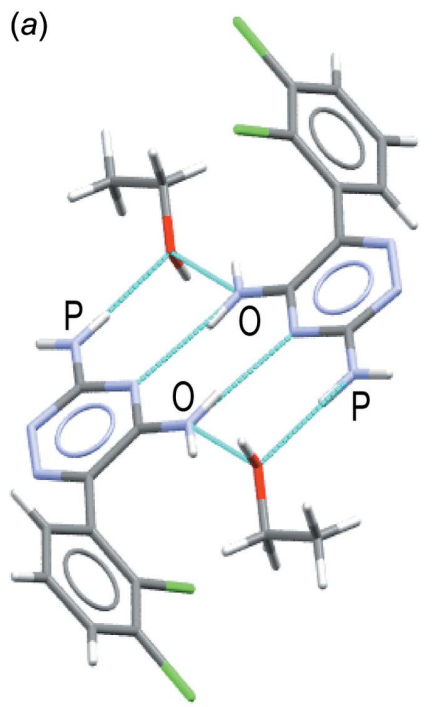

Current study

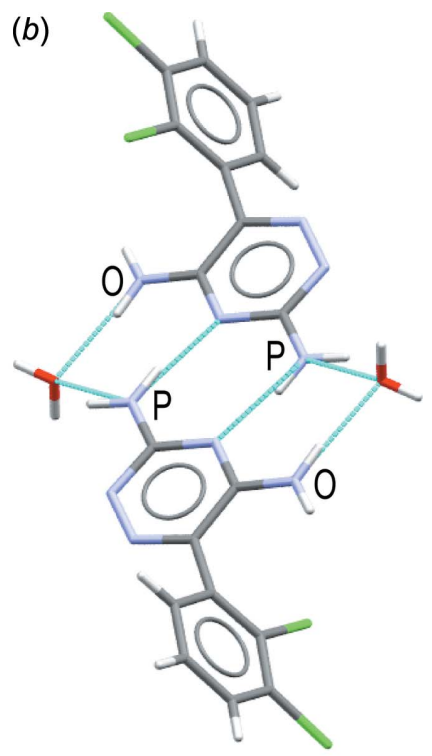

WUVLOP
Figure 4

(a) The dimerization motif in (I), held together with the amines in the ortho position of the triazine group. The amine in the ortho and para positions are labelled with $\mathrm{O}$ and $\mathrm{P}$, respectively. $(b)$ The dimerization motif in the ethanolate hydrate structure, held together with the amines in the para position of the triazine group.
Table 3

Experimental details.

\begin{tabular}{|c|c|}
\hline \multicolumn{2}{|l|}{ Crystal data } \\
\hline Chemical formula & $\mathrm{C}_{9} \mathrm{H}_{7} \mathrm{Cl}_{2} \mathrm{~N}_{5} \cdot \mathrm{C}_{2} \mathrm{H}_{6} \mathrm{O}$ \\
\hline$M_{\mathrm{r}}$ & 302.16 \\
\hline Crystal system, space group & Monoclinic, $C 2 / c$ \\
\hline Temperature (K) & 100 \\
\hline$a, b, c(\AA)$ & $\begin{array}{l}21.2458(15), 10.2320(8), \\
\quad 14.8428(11)\end{array}$ \\
\hline$\beta\left({ }^{\circ}\right)$ & $118.808(4)$ \\
\hline$V\left(\AA^{6}\right)$ & $2827.3(4)$ \\
\hline$Z$ & 8 \\
\hline Radiation type & Мо $K \alpha$ \\
\hline$\mu\left(\mathrm{mm}^{-1}\right)$ & 0.46 \\
\hline Crystal size $(\mathrm{mm})$ & $0.39 \times 0.25 \times 0.13$ \\
\hline
\end{tabular}

Data collection

Diffractometer

Absorption correction

$T_{\min }, T_{\max }$

No. of measured, independent and observed $[I>2 \sigma(I)]$ reflections

$R_{\text {int }}$

$(\sin \theta / \lambda)_{\max }\left(\AA^{-1}\right)$

Bruker APEXII CCD
Multi-scan (SADABS; Bruker,
2015)
$0.602,0.745$
$21376,2925,2634$

0.053
0.629

$0.098,0.234,1.41$
2925
193
48
H-atom parameters constrained
$0.62,-0.87$

Refinement

$R\left[F^{2}>2 \sigma\left(F^{2}\right)\right], w R\left(F^{2}\right), S$

No. of reflections

No. of parameters

No. of restraints

$\mathrm{H}$-atom treatment

$\Delta \rho_{\max }, \Delta \rho_{\min }\left(\mathrm{e} \AA^{-3}\right)$

$\mathrm{H}$-atom parameters constrained

Computer programs: APEX2 and SAINT (Bruker, 2015), SHELXT (Sheldrick, 2015a), SHELXL (Sheldrick, 2015b) and Olex2 (Dolomanov et al., 2009).

\section{Synthesis and crystallization}

Lamotrigine ( $>98 \%$, Acros Organics) was saturated in a solution of pure anhydrous ethanol (>99.5\%, Sigma Aldrich) over several weeks. Crystals of lamotrigine ethanolate were produced via slow evaporation of $1 \mathrm{ml}$ of the solution over $72 \mathrm{~h}$.

\section{Refinement}

Crystal data, data collection and structure refinement details are summarized in Table 3. All of the hydrogen atoms were located geometrically (aromatic $\mathrm{C}-\mathrm{H}=0.95 \AA$, methyl $\mathrm{C}-\mathrm{H}$ $=0.98 \AA$, ethyl $\mathrm{C}-\mathrm{H}=0.99 \AA, \mathrm{O}-\mathrm{H}=0.84 \AA \mathrm{N}-\mathrm{H}=0.88 \AA$ ) and refined using a riding model [aromatic, ethyl and amine $U_{\text {iso }}(\mathrm{H})=1.2$ times parent atom $U_{\text {eq }}$, methyl and alcohol $U_{\text {iso }}(\mathrm{H})=1.5$ times parent atom $U_{\text {eq }}$. The ethanol solvent in the lattice is disordered over two positions; the occupancies of the two positions were refined with the sum set to equal 1, refining to give relative occupancies of 52:48. Restraints (SIMU 0.010 .02 ) were applied to maintain sensible thermal displacement parameters for the carbon atoms.

\section{Funding information}

SRH, CLH and JP would like to thank MagnaPharm, a collaborative research project funded by the European Union's Horizon 2020 Research and Innovation programme 
(grant No. 736899), the Bristol Centre for Functional Nanomaterials (EP/G036780/1) and the Centre for Doctoral Training in Condensed Matter Physics (EP/L015544/1).

\section{References}

Bruker (2015). APEX2, SAINT and SADABS. Bruker AXS Inc., Madison, Wisconsin USA.

Cheney, M. L., Shan, N., Healey, E. R., Hanna, M., Wojtas, L., Zaworotko, M. J., Sava, V., Song, S. \& Sanchez-Ramos, J. R. (2010). Cryst. Growth Des. 10, 394-405.

Dolomanov, O. V., Bourhis, L. J., Gildea, R. J., Howard, J. A. K. \& Puschmann, H. (2009). J. Appl. Cryst. 42, 339-341.

Garti, N., Berkovich, Y., Dolitzky, B. Z., Aronhime, J., Singer, C., Liebermann, A. \& Gershon, N. (2008). US Patent Number. 7390807B2.
Groom, C. R., Bruno, I. J., Lightfoot, M. P. \& Ward, S. C. (2016). Acta Cryst. B72, 171-179.

Hanna, M., Shan, N. \& Cheney, M. L. (2009). US Patent Number. 061513A1.

Janes, R. W., Lisgarten, J. N. \& Palmer, R. A. (1989). Acta Cryst. C45, 129-132.

Qian, Y., Lv, P. C., Shi, L., Fang, R. Q., Song, Z. C. \& Zhu, H. L. (2009). J. Chem. Sci. 121, 463-470.

Sheldrick, G. M. (2015a). Acta Cryst. A71, 3-8.

Sheldrick, G. M. (2015b). Acta Cryst. C71, 3-8.

Sridhar, S. \& Ravikumar, K. (2011). J. Chem. Crystallogr. 41, 1289 1300.

Uzunović, A., Vranić, E. \& Hadžidedić, Š. (2010). Bosn. J. Basic Med. Sci. 10, 234-238.

Vaithianathan, A., Raman, S., Jiang, W., Ting, Y. T., Kane, M. A. \& Polli, J. E. (2015). Mol. Pharm. 12, 2436-2443.

Widanapathirana, L., Tale, S. \& Reineke, T. M. (2015). Mol. Pharm. 12, 2537-2543. 


\section{supporting information}

Acta Cryst. (2018). E74, 678-681［https://doi.org/10.1107/S2056989018005819]

\section{Lamotrigine ethanol monosolvate}

\section{Charlie L. Hall, Jason Potticary, Hazel A. Sparkes, Natalie E. Pridmore and Simon R. Hall}

Computing details

Data collection: APEX2 (Bruker, 2015); cell refinement: SAINT (Bruker, 2015); data reduction: SAINT (Bruker, 2015); program(s) used to solve structure: SHELXT (Sheldrick, 2015a); program(s) used to refine structure: SHELXL (Sheldrick, 2015b); molecular graphics: Olex2 (Dolomanov et al., 2009); software used to prepare material for publication: Olex2 (Dolomanov et al., 2009).

(I)

Crystal data

$\mathrm{C}_{9} \mathrm{H}_{7} \mathrm{Cl}_{2} \mathrm{~N}_{5} \cdot \mathrm{C}_{2} \mathrm{H}_{6} \mathrm{O}$

$M_{r}=302.16$

Monoclinic, $C 2 / c$

$a=21.2458(15) \AA$

$b=10.2320(8) \AA$

$c=14.8428(11) \AA$

$\beta=118.808(4)^{\circ}$

$V=2827.3(4) \AA^{3}$

$Z=8$

Data collection

Bruker APEXII CCD diffractometer

Radiation source: fine-focus sealed tube

Graphite monochromator

$\varphi$ and $\omega$ scans

Absorption correction: multi-scan

(SADABS; Bruker, 2015)

$T_{\text {min }}=0.602, T_{\text {max }}=0.745$

Refinement

Refinement on $F^{2}$

Least-squares matrix: full

$R\left[F^{2}>2 \sigma\left(F^{2}\right)\right]=0.098$

$w R\left(F^{2}\right)=0.234$

$S=1.41$

2925 reflections

193 parameters

48 restraints

Primary atom site location: dual
$F(000)=1248$

$D_{\mathrm{x}}=1.420 \mathrm{Mg} \mathrm{m}^{-3}$

Mo $K \alpha$ radiation, $\lambda=0.71073 \AA$

Cell parameters from 7221 reflections

$\theta=2.2-26.4^{\circ}$

$\mu=0.46 \mathrm{~mm}^{-1}$

$T=100 \mathrm{~K}$

Block, colourless

$0.39 \times 0.25 \times 0.13 \mathrm{~mm}$

21376 measured reflections

2925 independent reflections

2634 reflections with $I>2 \sigma(I)$

$R_{\text {int }}=0.053$

$\theta_{\text {max }}=26.6^{\circ}, \theta_{\text {min }}=2.2^{\circ}$

$h=-26 \rightarrow 26$

$k=-12 \rightarrow 12$

$l=-18 \rightarrow 18$

Hydrogen site location: inferred from neighbouring sites

$\mathrm{H}$-atom parameters constrained

$w=1 /\left[\sigma^{2}\left(F_{\mathrm{o}}^{2}\right)+59.8676 P\right]$

where $P=\left(F_{\mathrm{o}}^{2}+2 F_{\mathrm{c}}{ }^{2}\right) / 3$

$(\Delta / \sigma)_{\max }<0.001$

$\Delta \rho_{\max }=0.62 \mathrm{e} \AA^{-3}$

$\Delta \rho_{\min }=-0.87$ e $\AA^{-3}$ 


\section{Special details}

Geometry. All esds (except the esd in the dihedral angle between two 1.s. planes) are estimated using the full covariance matrix. The cell esds are taken into account individually in the estimation of esds in distances, angles and torsion angles; correlations between esds in cell parameters are only used when they are defined by crystal symmetry. An approximate (isotropic) treatment of cell esds is used for estimating esds involving l.s. planes.

Refinement. The occupancies of the disordered atoms in the ethanol were refined with their sum set to equal 1 . Restraints were applied to maintain sensible thermal and geometric parameters. The diffraction data showed slight splitting of some peaks but twinning could not be sensibly separated and modelled. However this may explain the large K values, slightly high second weight paramater and Fobs greater than Fcalc.

Fractional atomic coordinates and isotropic or equivalent isotropic displacement parameters $\left(\AA^{2}\right)$

\begin{tabular}{|c|c|c|c|c|c|}
\hline & $x$ & $y$ & $z$ & $U_{\text {iso }} * / U_{\text {eq }}$ & Occ. $(<1)$ \\
\hline $\mathrm{Cl1}$ & $0.16117(9)$ & $0.41684(18)$ & $0.10486(12)$ & 0.0254 (4) & \\
\hline $\mathrm{Cl} 2$ & $0.06655(8)$ & $0.37527(18)$ & $0.21033(13)$ & $0.0256(4)$ & \\
\hline $\mathrm{O} 1$ & $0.4106(2)$ & $0.2925(5)$ & $0.4111(3)$ & $0.0192(10)$ & \\
\hline H1A & 0.397808 & 0.357810 & 0.371958 & $0.029^{*}$ & 0.484 (14) \\
\hline H1B & 0.398027 & 0.358121 & 0.372284 & $0.029 *$ & $0.516(14)$ \\
\hline N3 & $0.3251(3)$ & $0.6534(5)$ & 0.1015 (4) & $0.0136(10)$ & \\
\hline N4 & $0.2195(3)$ & $0.7095(5)$ & 0.0967 (4) & $0.0145(11)$ & \\
\hline $\mathrm{H} 4 \mathrm{~A}$ & 0.208617 & 0.757236 & 0.041803 & $0.017^{*}$ & \\
\hline $\mathrm{H} 4 \mathrm{~B}$ & 0.189512 & 0.705023 & 0.121767 & $0.017^{*}$ & \\
\hline N1 & $0.3666(3)$ & $0.5147(6)$ & $0.2792(4)$ & $0.0177(11)$ & \\
\hline $\mathrm{N} 2$ & $0.4130(3)$ & $0.5288(6)$ & $0.2425(4)$ & $0.0185(12)$ & \\
\hline N5 & $0.4347(3)$ & $0.6032(7)$ & $0.1152(4)$ & $0.0293(15)$ & \\
\hline $\mathrm{H} 5 \mathrm{~A}$ & 0.422053 & 0.646160 & 0.057650 & $0.035^{*}$ & \\
\hline $\mathrm{H} 5 \mathrm{~B}$ & 0.477226 & 0.565850 & 0.147585 & $0.035^{*}$ & \\
\hline C6 & $0.2549(3)$ & $0.5427(6)$ & $0.2790(4)$ & $0.0144(12)$ & \\
\hline $\mathrm{C} 8$ & $0.2808(3)$ & $0.6445(6)$ & 0.1415 (4) & $0.0133(12)$ & \\
\hline $\mathrm{C} 7$ & $0.3022(3)$ & $0.5648(6)$ & $0.2321(4)$ & $0.0142(12)$ & \\
\hline $\mathrm{C} 4$ & $0.2351(4)$ & 0.5649 (7) & $0.4253(5)$ & $0.0191(13)$ & \\
\hline $\mathrm{H} 4$ & 0.251372 & 0.593442 & 0.493888 & $0.023^{*}$ & \\
\hline $\mathrm{C} 3$ & $0.1697(3)$ & $0.5029(7)$ & $0.3727(5)$ & $0.0193(14)$ & \\
\hline H3 & 0.140279 & 0.491009 & 0.404086 & $0.023^{*}$ & \\
\hline $\mathrm{C} 2$ & $0.1470(3)$ & $0.4581(7)$ & $0.2738(5)$ & $0.0176(13)$ & \\
\hline C9 & $0.3894(3)$ & $0.5948(7)$ & $0.1536(5)$ & $0.0189(13)$ & \\
\hline $\mathrm{C} 5$ & $0.2772(3)$ & $0.5859(7)$ & $0.3788(5)$ & $0.0188(13)$ & \\
\hline H5 & 0.321851 & 0.630185 & 0.415469 & $0.023^{*}$ & \\
\hline $\mathrm{C} 1$ & $0.1893(3)$ & $0.4782(6)$ & $0.2273(5)$ & $0.0152(12)$ & \\
\hline $\mathrm{C} 10 \mathrm{~B}$ & $0.4570(8)$ & $0.2138(16)$ & $0.3909(13)$ & $0.024(3)$ & $0.484(14)$ \\
\hline $\mathrm{H} 10 \mathrm{~A}$ & 0.435281 & 0.197369 & 0.316042 & $0.029 *$ & $0.484(14)$ \\
\hline H10B & 0.464411 & 0.128524 & 0.426119 & $0.029 *$ & $0.484(14)$ \\
\hline C11B & $0.5275(8)$ & $0.2819(16)$ & $0.4283(14)$ & $0.030(4)$ & $0.484(14)$ \\
\hline H11A & 0.519935 & 0.365998 & 0.393000 & $0.045^{*}$ & $0.484(14)$ \\
\hline H11B & 0.559689 & 0.227634 & 0.414026 & $0.045^{*}$ & $0.484(14)$ \\
\hline $\mathrm{H} 11 \mathrm{C}$ & 0.549146 & 0.296685 & 0.502588 & $0.045^{*}$ & $0.484(14)$ \\
\hline $\mathrm{C} 10 \mathrm{~A}$ & $0.4866(8)$ & $0.2617(17)$ & $0.4439(12)$ & 0.030 & $0.516(14)$ \\
\hline $\mathrm{H} 10 \mathrm{C}$ & 0.500914 & 0.183860 & 0.489087 & $0.036^{*}$ & $0.516(14)$ \\
\hline
\end{tabular}




$\begin{array}{llllll}\text { H10D } & 0.516983 & 0.335764 & 0.484496 & 0.036^{*} & 0.516(14) \\ \text { C11A } & 0.5000(8) & 0.2356(15) & 0.3545(12) & 0.030(3) & 0.516(14) \\ \text { H11D } & 0.466604 & 0.168389 & 0.310075 & 0.045^{*} & 0.516(14) \\ \text { H11E } & 0.549503 & 0.205101 & 0.380438 & 0.045^{*} & 0.516(14) \\ \text { H11F } & 0.492715 & 0.316205 & 0.315082 & 0.045^{*} & 0.516(14)\end{array}$

Atomic displacement parameters $\left(\AA^{2}\right)$

\begin{tabular}{|c|c|c|c|c|c|c|}
\hline & $U^{11}$ & $U^{22}$ & $U^{33}$ & $U^{12}$ & $U^{13}$ & $U^{23}$ \\
\hline $\mathrm{Cl1}$ & $0.0342(9)$ & $0.0289(9)$ & $0.0201(8)$ & $-0.0150(7)$ & $0.0189(7)$ & $-0.0098(7)$ \\
\hline $\mathrm{Cl} 2$ & $0.0144(7)$ & $0.0370(10)$ & $0.0260(8)$ & $-0.0077(7)$ & $0.0102(6)$ & $0.0040(7)$ \\
\hline $\mathrm{O} 1$ & $0.017(2)$ & $0.024(2)$ & $0.020(2)$ & $0.0066(18)$ & 0.0105 (18) & 0.0100 (19) \\
\hline N3 & $0.010(2)$ & 0.018 & $0.011(2)$ & $0.003(2)$ & $0.004(2)$ & $0.004(2)$ \\
\hline N4 & $0.011(2)$ & $0.020(3)$ & $0.012(2)$ & $0.005(2)$ & $0.006(2)$ & $0.007(2)$ \\
\hline N1 & $0.016(3)$ & $0.023(3)$ & $0.016(3)$ & $0.001(2)$ & $0.010(2)$ & $0.003(2)$ \\
\hline N2 & $0.010(2)$ & $0.031(3)$ & $0.015(3)$ & $0.006(2)$ & $0.007(2)$ & $0.008(2)$ \\
\hline N5 & $0.017(3)$ & $0.056(4)$ & $0.019(3)$ & $0.017(3)$ & $0.012(2)$ & 0.018 \\
\hline C6 & $0.015(3)$ & $0.016(3)$ & $0.013(3)$ & $0.005(2)$ & $0.008(2)$ & $0.004(2)$ \\
\hline $\mathrm{C} 8$ & $0.014(3)$ & $0.015(3)$ & $0.012(3)$ & $0.000(2)$ & $0.007(2)$ & $-0.001(2)$ \\
\hline $\mathrm{C} 7$ & $0.009(3)$ & $0.019(3)$ & $0.014(3)$ & $0.000(2)$ & $0.006(2)$ & $0.000(2)$ \\
\hline $\mathrm{C} 4$ & $0.025(3)$ & $0.020(3)$ & $0.013(3)$ & $0.003(3)$ & $0.010(3)$ & $0.003(3)$ \\
\hline $\mathrm{C} 3$ & $0.021(3)$ & $0.022(3)$ & $0.023(3)$ & $0.009(3)$ & $0.018(3)$ & 0.009 (3) \\
\hline $\mathrm{C} 2$ & $0.014(3)$ & $0.021(3)$ & $0.019(3)$ & -0.001 & 0.009 (3) & $0.002(3)$ \\
\hline C9 & $0.015(3)$ & $0.027(4)$ & $0.017(3)$ & $0.008(3)$ & $0.010(2)$ & $0.008(3)$ \\
\hline $\mathrm{C} 5$ & $0.015(3)$ & $0.024(3)$ & $0.015(3)$ & $0.002(3)$ & $0.006(2)$ & $0.004(3)$ \\
\hline $\mathrm{C} 1$ & $0.019(3)$ & $0.015(3)$ & $0.012(3)$ & $0.003(2)$ & 0.009 (2) & $0.002(2)$ \\
\hline C10B & $0.020(5)$ & $0.026(5)$ & $0.028(5)$ & $0.006(5)$ & $0.013(4)$ & $0.005(5)$ \\
\hline C11B & $0.020(7)$ & $0.028(7)$ & $0.044(8)$ & $0.008(6)$ & $0.017(6)$ & $0.015(6)$ \\
\hline C10A & $0.017(5)$ & $0.039(6)$ & $0.031(5)$ & $0.007(5)$ & $0.011(4)$ & $0.013(5)$ \\
\hline C11A & $0.030(6)$ & $0.027(7)$ & $0.040(7)$ & $0.009(6)$ & $0.021(6)$ & $0.008(6)$ \\
\hline
\end{tabular}

Geometric parameters $\left(\AA,{ }^{\circ}\right)$

\begin{tabular}{llll}
\hline $\mathrm{C} 11-\mathrm{C} 1$ & $1.735(6)$ & $\mathrm{C} 8-\mathrm{C} 7$ & $1.446(8)$ \\
$\mathrm{C} 12-\mathrm{C} 2$ & $1.725(6)$ & $\mathrm{C} 4-\mathrm{H} 4$ & 0.9500 \\
$\mathrm{O} 1-\mathrm{H} 1 \mathrm{~A}$ & 0.8400 & $\mathrm{C} 4-\mathrm{C} 3$ & $1.377(9)$ \\
$\mathrm{O} 1-\mathrm{H} 1 \mathrm{~B}$ & 0.8400 & $\mathrm{C} 4-\mathrm{C} 5$ & $1.386(9)$ \\
$\mathrm{O} 1-\mathrm{C} 10 \mathrm{~B}$ & $1.413(15)$ & $\mathrm{C} 3-\mathrm{H} 3$ & 0.9500 \\
$\mathrm{O} 1-\mathrm{C} 10 \mathrm{~A}$ & $1.477(14)$ & $\mathrm{C} 3-\mathrm{C} 2$ & $1.384(9)$ \\
$\mathrm{N} 3-\mathrm{C} 8$ & $1.335(7)$ & $\mathrm{C} 2-\mathrm{C} 1$ & $1.387(8)$ \\
$\mathrm{N} 3-\mathrm{C} 9$ & $1.344(8)$ & $\mathrm{C} 5-\mathrm{H} 5$ & 0.9500 \\
$\mathrm{~N} 4-\mathrm{H} 4 \mathrm{~A}$ & 0.8800 & $\mathrm{C} 10 \mathrm{~B}-\mathrm{H} 10 \mathrm{~A}$ & 0.9900 \\
$\mathrm{~N} 4-\mathrm{H} 4 \mathrm{~B}$ & 0.8800 & $\mathrm{C} 10 \mathrm{~B}-\mathrm{H} 10 \mathrm{~B}$ & 0.9900 \\
$\mathrm{~N} 4-\mathrm{C} 8$ & $1.322(8)$ & $\mathrm{C} 10 \mathrm{~B}-\mathrm{C} 11 \mathrm{~B}$ & $1.50(2)$ \\
$\mathrm{N} 1-\mathrm{N} 2$ & $1.345(7)$ & $\mathrm{C} 11 \mathrm{~B}-\mathrm{H} 11 \mathrm{~A}$ & 0.9800 \\
$\mathrm{~N} 1-\mathrm{C} 7$ & $1.304(8)$ & $\mathrm{C} 11 \mathrm{~B}-\mathrm{H} 11 \mathrm{~B}$ & 0.9800 \\
$\mathrm{~N} 2-\mathrm{C} 9$ & $1.346(8)$ & $\mathrm{C} 11 \mathrm{~B}-\mathrm{H} 11 \mathrm{C}$ & 0.9800 \\
$\mathrm{~N} 5-\mathrm{H} 5 \mathrm{C}$ & 0.8800 & $\mathrm{C} 10 \mathrm{~A}-\mathrm{H} 10 \mathrm{C}$ & 0.9900
\end{tabular}




\begin{tabular}{|c|c|c|c|}
\hline N5-H5B & 0.8800 & $\mathrm{C} 10 \mathrm{~A}-\mathrm{H} 10 \mathrm{D}$ & 0.9900 \\
\hline $\mathrm{N} 5-\mathrm{C} 9$ & $1.336(8)$ & $\mathrm{C} 10 \mathrm{~A}-\mathrm{C} 11 \mathrm{~A}$ & $1.51(2)$ \\
\hline $\mathrm{C} 6-\mathrm{C} 7$ & $1.490(8)$ & $\mathrm{C} 11 \mathrm{~A}-\mathrm{H} 11 \mathrm{D}$ & 0.9800 \\
\hline $\mathrm{C} 6-\mathrm{C} 5$ & $1.392(9)$ & $\mathrm{C} 11 \mathrm{~A}-\mathrm{H} 11 \mathrm{E}$ & 0.9800 \\
\hline $\mathrm{C} 6-\mathrm{C} 1$ & $1.391(9)$ & $\mathrm{C} 11 \mathrm{~A}-\mathrm{H} 11 \mathrm{~F}$ & 0.9800 \\
\hline $\mathrm{C} 10 \mathrm{~B}-\mathrm{O} 1-\mathrm{H} 1 \mathrm{~A}$ & 109.5 & $\mathrm{~N} 5-\mathrm{C} 9-\mathrm{N} 2$ & $116.5(6)$ \\
\hline $\mathrm{C} 10 \mathrm{~A}-\mathrm{O} 1-\mathrm{H} 1 \mathrm{~B}$ & 109.5 & $\mathrm{C} 6-\mathrm{C} 5-\mathrm{H} 5$ & 119.7 \\
\hline $\mathrm{C} 8-\mathrm{N} 3-\mathrm{C} 9$ & $116.9(5)$ & $\mathrm{C} 4-\mathrm{C} 5-\mathrm{C} 6$ & $120.6(6)$ \\
\hline $\mathrm{H} 4 \mathrm{~A}-\mathrm{N} 4-\mathrm{H} 4 \mathrm{~B}$ & 120.0 & $\mathrm{C} 4-\mathrm{C} 5-\mathrm{H} 5$ & 119.7 \\
\hline $\mathrm{C} 8-\mathrm{N} 4-\mathrm{H} 4 \mathrm{~A}$ & 120.0 & $\mathrm{C} 6-\mathrm{C} 1-\mathrm{Cl1}$ & $119.8(5)$ \\
\hline $\mathrm{C} 8-\mathrm{N} 4-\mathrm{H} 4 \mathrm{~B}$ & 120.0 & $\mathrm{C} 2-\mathrm{C} 1-\mathrm{Cl} 1$ & $119.2(5)$ \\
\hline $\mathrm{C} 7-\mathrm{N} 1-\mathrm{N} 2$ & $121.7(5)$ & $\mathrm{C} 2-\mathrm{C} 1-\mathrm{C} 6$ & $120.9(6)$ \\
\hline $\mathrm{N} 1-\mathrm{N} 2-\mathrm{C} 9$ & $116.9(5)$ & $\mathrm{O} 1-\mathrm{C} 10 \mathrm{~B}-\mathrm{H} 10 \mathrm{~A}$ & 109.8 \\
\hline $\mathrm{H} 5 \mathrm{~A}-\mathrm{N} 5-\mathrm{H} 5 \mathrm{~B}$ & 120.0 & $\mathrm{O} 1-\mathrm{C} 10 \mathrm{~B}-\mathrm{H} 10 \mathrm{~B}$ & 109.8 \\
\hline C9-N5-H5A & 120.0 & $\mathrm{O} 1-\mathrm{C} 10 \mathrm{~B}-\mathrm{C} 11 \mathrm{~B}$ & $109.3(13)$ \\
\hline $\mathrm{C} 9-\mathrm{N} 5-\mathrm{H} 5 \mathrm{~B}$ & 120.0 & $\mathrm{H} 10 \mathrm{~A}-\mathrm{C} 10 \mathrm{~B}-\mathrm{H} 10 \mathrm{~B}$ & 108.3 \\
\hline $\mathrm{C} 5-\mathrm{C} 6-\mathrm{C} 7$ & $119.2(6)$ & $\mathrm{C} 11 \mathrm{~B}-\mathrm{C} 10 \mathrm{~B}-\mathrm{H} 10 \mathrm{~A}$ & 109.8 \\
\hline $\mathrm{C} 1-\mathrm{C} 6-\mathrm{C} 7$ & $122.4(5)$ & $\mathrm{C} 11 \mathrm{~B}-\mathrm{C} 10 \mathrm{~B}-\mathrm{H} 10 \mathrm{~B}$ & 109.8 \\
\hline $\mathrm{C} 1-\mathrm{C} 6-\mathrm{C} 5$ & $118.3(6)$ & $\mathrm{C} 10 \mathrm{~B}-\mathrm{C} 11 \mathrm{~B}-\mathrm{H} 11 \mathrm{~A}$ & 109.5 \\
\hline $\mathrm{N} 3-\mathrm{C} 8-\mathrm{C} 7$ & $118.6(5)$ & $\mathrm{C} 10 \mathrm{~B}-\mathrm{C} 11 \mathrm{~B}-\mathrm{H} 11 \mathrm{~B}$ & 109.5 \\
\hline $\mathrm{N} 4-\mathrm{C} 8-\mathrm{N} 3$ & $118.5(5)$ & $\mathrm{C} 10 \mathrm{~B}-\mathrm{C} 11 \mathrm{~B}-\mathrm{H} 11 \mathrm{C}$ & 109.5 \\
\hline $\mathrm{N} 4-\mathrm{C} 8-\mathrm{C} 7$ & $122.9(5)$ & $\mathrm{H} 11 \mathrm{~A}-\mathrm{C} 11 \mathrm{~B}-\mathrm{H} 11 \mathrm{~B}$ & 109.5 \\
\hline $\mathrm{N} 1-\mathrm{C} 7-\mathrm{C} 6$ & $117.3(5)$ & $\mathrm{H} 11 \mathrm{~A}-\mathrm{C} 11 \mathrm{~B}-\mathrm{H} 11 \mathrm{C}$ & 109.5 \\
\hline $\mathrm{N} 1-\mathrm{C} 7-\mathrm{C} 8$ & $119.9(5)$ & $\mathrm{H} 11 \mathrm{~B}-\mathrm{C} 11 \mathrm{~B}-\mathrm{H} 11 \mathrm{C}$ & 109.5 \\
\hline $\mathrm{C} 8-\mathrm{C} 7-\mathrm{C} 6$ & $122.7(5)$ & $\mathrm{O} 1-\mathrm{C} 10 \mathrm{~A}-\mathrm{H} 10 \mathrm{C}$ & 109.0 \\
\hline $\mathrm{C} 3-\mathrm{C} 4-\mathrm{H} 4$ & 119.8 & $\mathrm{O} 1-\mathrm{C} 10 \mathrm{~A}-\mathrm{H} 10 \mathrm{D}$ & 109.0 \\
\hline $\mathrm{C} 3-\mathrm{C} 4-\mathrm{C} 5$ & $120.5(6)$ & $\mathrm{O} 1-\mathrm{C} 10 \mathrm{~A}-\mathrm{C} 11 \mathrm{~A}$ & $112.8(12)$ \\
\hline $\mathrm{C} 5-\mathrm{C} 4-\mathrm{H} 4$ & 119.8 & $\mathrm{H} 10 \mathrm{C}-\mathrm{C} 10 \mathrm{~A}-\mathrm{H} 10 \mathrm{D}$ & 107.8 \\
\hline $\mathrm{C} 4-\mathrm{C} 3-\mathrm{H} 3$ & 120.2 & $\mathrm{C} 11 \mathrm{~A}-\mathrm{C} 10 \mathrm{~A}-\mathrm{H} 10 \mathrm{C}$ & 109.0 \\
\hline $\mathrm{C} 4-\mathrm{C} 3-\mathrm{C} 2$ & $119.6(6)$ & $\mathrm{C} 11 \mathrm{~A}-\mathrm{C} 10 \mathrm{~A}-\mathrm{H} 10 \mathrm{D}$ & 109.0 \\
\hline $\mathrm{C} 2-\mathrm{C} 3-\mathrm{H} 3$ & 120.2 & $\mathrm{C} 10 \mathrm{~A}-\mathrm{C} 11 \mathrm{~A}-\mathrm{H} 11 \mathrm{D}$ & 109.5 \\
\hline $\mathrm{C} 3-\mathrm{C} 2-\mathrm{C} 12$ & $119.3(5)$ & $\mathrm{C} 10 \mathrm{~A}-\mathrm{C} 11 \mathrm{~A}-\mathrm{H} 11 \mathrm{E}$ & 109.5 \\
\hline $\mathrm{C} 3-\mathrm{C} 2-\mathrm{C} 1$ & $120.0(6)$ & $\mathrm{C} 10 \mathrm{~A}-\mathrm{C} 11 \mathrm{~A}-\mathrm{H} 11 \mathrm{~F}$ & 109.5 \\
\hline $\mathrm{C} 1-\mathrm{C} 2-\mathrm{Cl} 2$ & $120.7(5)$ & $\mathrm{H} 11 \mathrm{D}-\mathrm{C} 11 \mathrm{~A}-\mathrm{H} 11 \mathrm{E}$ & 109.5 \\
\hline $\mathrm{N} 3-\mathrm{C} 9-\mathrm{N} 2$ & $125.6(5)$ & $\mathrm{H} 11 \mathrm{D}-\mathrm{C} 11 \mathrm{~A}-\mathrm{H} 11 \mathrm{~F}$ & 109.5 \\
\hline $\mathrm{N} 5-\mathrm{C} 9-\mathrm{N} 3$ & $117.9(6)$ & $\mathrm{H} 11 \mathrm{E}-\mathrm{C} 11 \mathrm{~A}-\mathrm{H} 11 \mathrm{~F}$ & 109.5 \\
\hline
\end{tabular}

Hydrogen-bond geometry $\left(\AA,{ }^{\circ}\right)$

\begin{tabular}{lllll}
\hline$D-\mathrm{H} \cdots A$ & $D-\mathrm{H}$ & $\mathrm{H} \cdots A$ & $D \cdots A$ & $D-\mathrm{H} \cdots A$ \\
\hline $\mathrm{O} 1-\mathrm{H} 1 A \cdots \mathrm{N} 1$ & 0.84 & 2.01 & $2.848(7)$ & 179 \\
$\mathrm{~N} 4-\mathrm{H} 4 A \cdots \mathrm{N} 3^{\mathrm{i}}$ & 0.88 & 2.10 & $2.972(7)$ & 172 \\
$\mathrm{~N} 4-\mathrm{H} 4 B \cdots \mathrm{O} 1^{\mathrm{ii}}$ & 0.88 & 2.14 & $2.841(7)$ & 137 \\
$\mathrm{~N} 5-\mathrm{H} 5 A \cdots \mathrm{O} 1^{\mathrm{iii}}$ & 0.88 & 2.16 & $3.014(7)$ & 163 \\
$\mathrm{~N} 5-\mathrm{H} 5 B \cdots \mathrm{N} 2^{\text {iv }}$ & 0.88 & 2.14 & $2.987(8)$ & 161
\end{tabular}

Symmetry codes: (i) $-x+1 / 2,-y+3 / 2,-z$; (ii) $-x+1 / 2, y+1 / 2,-z+1 / 2$; (iii) $x,-y+1, z-1 / 2$; (iv) $-x+1, y,-z+1 / 2$. 